\title{
Erratum to: Cost-effectiveness analysis of once-yearly injection of zoledronic acid for the treatment of osteoporosis in Japan
}

\author{
K. Moriwaki ${ }^{1} \cdot$ M. Mouri ${ }^{2,3} \cdot$ H. Hagino $^{4}$
}

Published online: 21 March 2017

(C) International Osteoporosis Foundation and National Osteoporosis Foundation 2017

\section{Erratum to: Osteoporos Int}

DOI 10.1007/s00198-017-3973-8

In Table 2, the incorrect value of 66.00 was given for the category 70 years old, T-score -3.0 / BT (vs ZOL + BT) in the column showing 10-year probability (\%) of hip fracture, The correct value is 6.00 , as shown here in the corrected version of the table.

The online version of the original article can be found at http://dx.doi. org/10.1007/s00198-017-3973-8

\section{H. Hagino}

hagino@med.tottori-u.ac.jp

1 Department of Medical Statistics, Kobe Pharmaceutical University, 4-19-1, Motoyamakita, Higashinada, Kobe 658-8558, Japan

2 Global Health Research Coordinating Center, Kanagawa Academy of Science and Technology, KSP East 3F 309, 3-2-1, Sakado, Takatsu, Kawasaki, Kanagawa, Japan

3 Project Research Institutes, Comprehensive Research Organization, Waseda University, Tokyo 169-8050, Japan

4 School of Health Sciences, Faculty of Medicine, Tottori University, 86 Nishi-cho, Yonago, Tottori 683-8503, Japan 


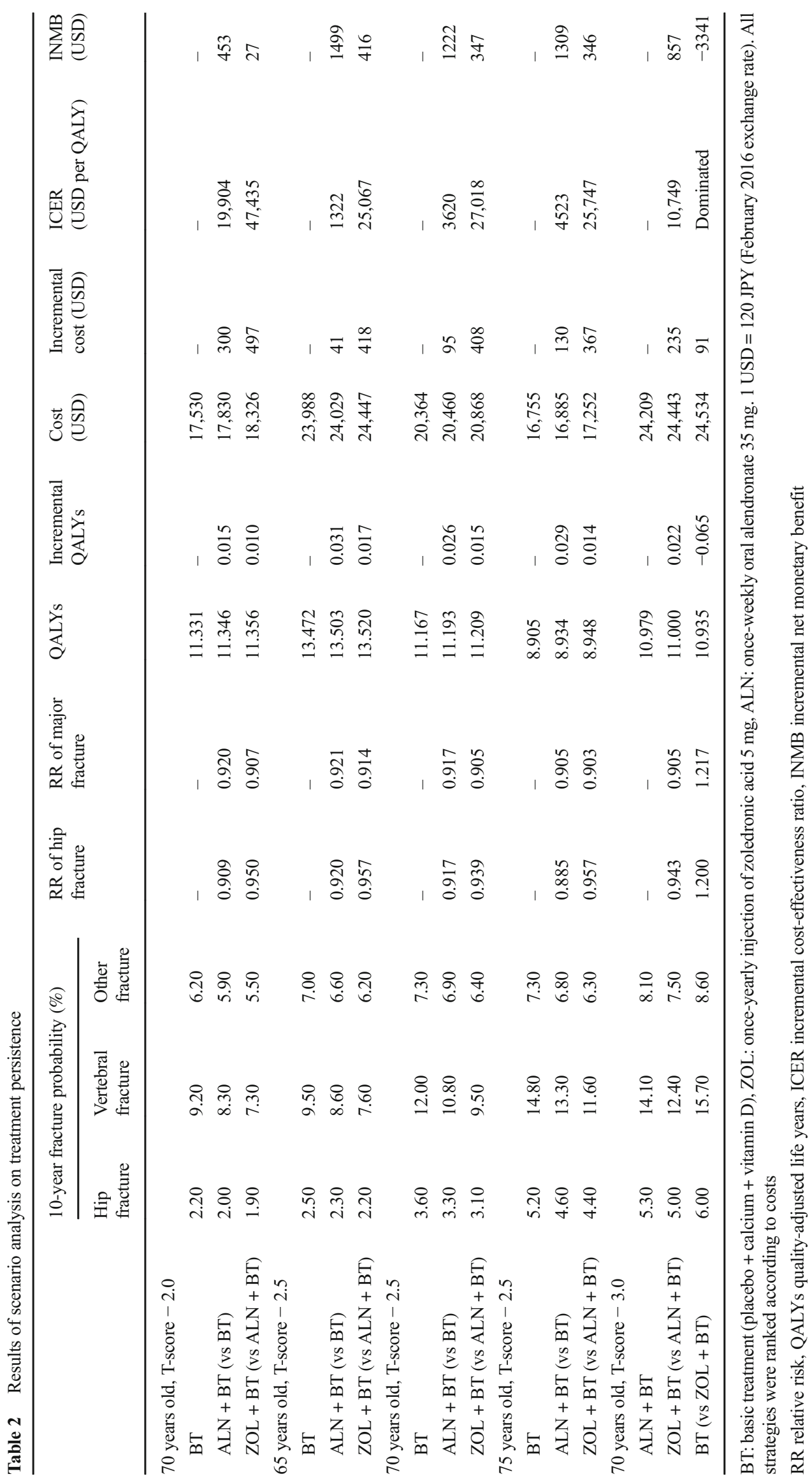

